\title{
MRI Characterization and Longitudinal Study of Focal Cerebellar Lesions in a Young Tuberous Sclerosis Cohort
}

\author{
J. Vaughn, M. Hagiwara, J. Katz, J. Roth, O. Devinsky, H. Weiner, and S. Milla
}

\begin{abstract}
BACKGROUND AND PURPOSE: There are few articles characterizing cerebellar lesions in patients with TSC and no published series documenting longitudinal evaluation of these lesions, to our knowledge. Recent suggestion of a correlation between autism and cerebellar lesions in patients with TSC heightens the importance of understanding these lesions. Our purpose was to characterize cerebellar lesions in a cohort of young patients with TSC with specific interest in assessing longitudinal changes.
\end{abstract}

MATERIALS AND METHODS: We retrospectively reviewed MR images from 145 pediatric and young adult patients with tuberous sclerosis (mean age, 7.6 years). A number of imaging characteristics of cerebellar tubers were recorded, and patients were evaluated for SGAs. Patients with follow-up scans $>3$ months from the original scan were further analyzed for longitudinal tuber characterization.

RESULTS: There were $24.1 \%$ of patients with focal cerebellar lesions; $52.4 \%$ of patients with cerebellar lesions demonstrated change in imaging characteristics during longitudinal analysis. Fifty-one percent of the lesions were enhanced after gadolinium administration. Twenty percent of the patients with cerebellar lesions had pathologically confirmed SGAs compared with the incidence of $11 \%$ in the 145 patients with TSC reviewed.

CONCLUSIONS: In our large cohort of young patients with TSC, cerebellar tubers were common and $52 \%$ of patients had tubers that changed with time. A higher percentage of patients with cerebellar lesions developed SGAs than patients with TSC without cerebellar lesions. Because this is the first reported longitudinal study of cerebellar lesions in TSC, further investigation may provide additional insight into TSC pathology and associated clinical manifestations, such as autism, developmental delay, and seizures.

ABBREVIATIONS: SENs = subependymal nodules; $\mathrm{SGAs}=$ subependymal giant cell astrocytomas; $\mathrm{TS}=$ tuberous sclerosis; TSC $=$ tuberous sclerosis complex

T uberous sclerosis complex is a genetic condition with a prevalence of approximately 1 in 6000 to 1 in 12,000 neonates. Two-thirds of the cases are sporadic, and one-third are inherited. ${ }^{1}$ The disorder is caused by mutations in the TSC1 or TSC2 tumorsuppressor genes with TSC2 mutations 3 times more common and associated with more severe symptoms. ${ }^{2}$ The disorder has a variable phenotype with lesions in multiple organs, including the skin and cardiac, vascular, central nervous, skeletal, and pulmonary and genitourinary systems.

Received April 25, 2012; accepted after revision June 11.

From the Department of Radiology (J.V., M.H., S.M.), Department of Neurosurgery (J.K.. H.W.), Division of Pediatric Neurosurgery, and Comprehensive Epilepsy Center (O.D.), New York University Langone Medical Center, New York, New York; and Department of Neurosurgery (J.R.), Tel Aviv Sourasky Medical Center, Tel Aviv, Israel.

Previously presented as an abstract at: Annual Meeting of the American Society of Neuroradiology, June 4-9, 2011; Seattle, Washington.

Please address correspondence to Jennifer Vaughn, MD, Department of Radiology, NYU Langone Medical Center, 550 First Ave, New York, NY 10016; e-mail:

Jennifer.Vaughn@nyumc.org

http://dx.doi.org/10.3174/ajnr.A3260
Most patients have central nervous system lesions, including supratentorial cortical tubers (often with subjacent white matter abnormalities), SENs, and SGAs. These central nervous system lesions are associated with high morbidity, including seizures in $75 \%-90 \%$ of patients, learning and developmental disabilities with subaverage intelligence in $\sim 50 \%$ of patients, ${ }^{3}$ and mild-tomoderate autistic spectrum disorders in $25 \%-50 \%$ of patients. Supratentorial cortical/subcortical tubers are present in $>90 \%$ of patients with TSC. Infratentorial lesions are not as well-studied and occur in $9 \%-44 \%$ of patients. ${ }^{4-6}$ A recent study found infratentorial lesions in one-third of 27 pediatric patients with TSC. ${ }^{7}$ Supratentorial tubers are typically thought to be stable lesions. However, a recent study demonstrated imaging change in 2 TSC patients out of 179 studied, with an increase in both size and number in pathologically proved cystlike tubers. ${ }^{8}$ Cellular proliferation in cortical tubers may explain the evolution and change in tuber size and appearance. ${ }^{9}$ One patient developed a new rightsided cerebellar lesion during a 3-year follow-up period suggesting evolution of the infratentorial tubers. ${ }^{10}$ Pathology revealed 
balloon cells, disorganized neuronal organization, and gliosis and calcification, similar to those features in supratentorial tubers. ${ }^{10}$ Thus, pathology specimens have suggested that these focal cerebellar lesions are likely tubers.

The purpose of our study was to determine the incidence of cerebellar abnormalities, particularly presumed tubers, in a large cohort of pediatric and young adult patients with TSC and to validate recent literature reports on the MR imaging characteristics of these lesions. The tubers were studied longitudinally to determine the presence, incidence, and nature of change in cerebellar tubers. A potential relationship between patients who develop SGAs and cerebellar tubers was also investigated.

\section{MATERIALS AND METHODS Study Design}

After institutional review board approval, we retrospectively searched for pediatric and young adult patients with TSC who underwent brain MR imaging from 1999 to 2010. A computeraided search by using the keywords "tuberous sclerosis," age constraints of $0-25$ years at the time of scanning, and study type "MR imaging brain" was performed to obtain radiologic reports. Inclusion criteria into our study group were a diagnosis of TSC confirmed by the presence of typical intracranial findings of TS and/or chart review, age at time of first scan of $0-25$ years, and PACS-available brain MR images with high-quality sequences. Exclusion criteria were age at the time of the first scan older than 25 years, uncertain tuberous sclerosis diagnosis, and poor-quality or incomplete images. Study subjects included 145 patients (72 males, 73 females; mean age, 7.6 years; and age range, $0-25$ years at the time of first scan).

\section{Imaging and Analysis}

Patients were scanned with 1.5T MR imaging, and standard departmental imaging protocols were used. Sequences included spin-echo T2WI, spin-echo T1WI, and FLAIR in all patients. 3D multiplanar reconstruction, gradient-echo $\mathrm{T} 2{ }^{*}$-weighted $(\mathrm{He}-$ moflash), DWI, and gadolinium-enhanced T1WI sequences were reviewed when available.

Scans were reviewed by 3 independent reviewers, including a radiology resident, a pediatric neuroradiologist, and a Certificate of Added Qualification-certified general neuroradiologist; consensus was obtained for all criteria examined when there was a difference in opinion on independent image review.

We identified all patients with TSC and reviewed all of their MRIs to determine whether cerebellar lesions were ever present. Imaging characteristics recorded included the following: number and location (side) of focal cerebellar lesions; signal characteristics of the lesions on T1, T2, and FLAIR sequences; the presence of lesion calcification; the presence of enhancement when gadolinium was administered; and evaluation for an associated retraction deformity. Cerebellar lesions were defined as intrinsic focal signal abnormality on at least 1 of the T1, T2, or FLAIR sequences, typically in a wedge- or bandlike distribution. The presence of calcification was inferred from low signal intensity on T2 sequences and susceptibility artifacts on gradient-echo sequences when available. Retraction deformity was defined as a focal contour abnormality at the periphery of the lesion with associated volume

\begin{tabular}{lc} 
Changes in lesions in patients with cerebellar tubers \\
\hline \multicolumn{1}{c}{ Nature of Lesion Change } & $\begin{array}{c}\text { Total No. of Lesions } \\
\text { Demonstrating This Change }\end{array}$ \\
\hline New lesion & 1 \\
Increased size & 5 \\
Decreased size & 2 \\
Increased/new calcification & 11 \\
Increased/new retraction deformity & 4 \\
Change in T2/FLAIR signal & 14 \\
Increased enhancement & 1 \\
New appearance of enhancement & 2 \\
Decreased enhancement & 2 \\
Disappearance of enhancement & 4 \\
\hline
\end{tabular}

loss. All cases were examined for global cerebellar atrophy and a history of resected SGA.

Patients with cerebellar lesions and follow-up brain MRI at $>3$ months from the initial scan were examined for longitudinal lesion characterization, including the number and location of new lesions and a change in existing lesion signal characteristics. Change in an individual lesion was defined as a change in size; T1, T2, or FLAIR signal; enhancement pattern; development or regression of calcification; and development of an associated retraction deformity.

\section{RESULTS}

Cerebellar tubers were identified in 35 of the 145 (24.1\%) patients, and a total of 54 cerebellar tubers were identified in these 35 patients. Twenty-six tubers were left-sided, and 28 were right-sided.

Signal characteristics of the tubers were the following: on FLAIR, 28 of 54 (51.9\%) were bright, 12 of 54 (22.2\%) were isointense, and 14 of 54 (25.9\%) were dark; on T1, 1 of 54 (1.9\%) was bright, 8 of $54(14.8 \%)$ were isointense, and 45 of 54 ( $88.3 \%)$ were dark; on T2, 47 of $54(87.0 \%)$ were bright and 7 of 54 (13.0\%) were dark. Gadolinium was administered to 31 of the 35 patients with tubers; in this group, there were 49 total tubers for evaluation of enhancement patterns. Twenty-five of these 49 tubers (51.0\%) enhanced on initial MR imaging. Calcification was present in 29 of the 54 (53.7\%) tubers. Retraction deformity associated with the tuber was noted in 46 of the 54 (85.2\%) tubers.

In 21 of the 35 patients, there was follow-up MR imaging with the range of follow-up among these patients varying from 3 months to 7 years (mean follow-up, 4.3 years). Eleven of these 21 patients $(52.4 \%)$ had at least 1 lesion that changed with time. In these 21 patients, there were 37 lesions, with 17 lesions changed $(45.9 \%)$. The average age of the patients with cerebellar tubers was 8.5 years, and the average age of those with lesions that changed was 6.4 years. The changes included new lesions $(n=1)$, change in lesion size $(n=7$; increased size $=5$, decreased size $=2)$, increased or new lesion calcification $(n=11)$, increased or new associated retraction deformity $(n=4)$, change in FLAIR and/or T2 signal $(n=14)$, and change in enhancement $(n=9$; new enhancement $=2$, increased enhancement $=1$, disappearance of enhancement $=4$, decreased enhancement $=2$ ) (Table and Figs $1-3)$. In these patients who had demonstrated changes in cerebellar lesions, the range of follow-up between the first and last scans was 11 months to 11 years (mean follow-up, 5.8 years).

In the patients with cerebellar tubers, 6 of the 35 patients 


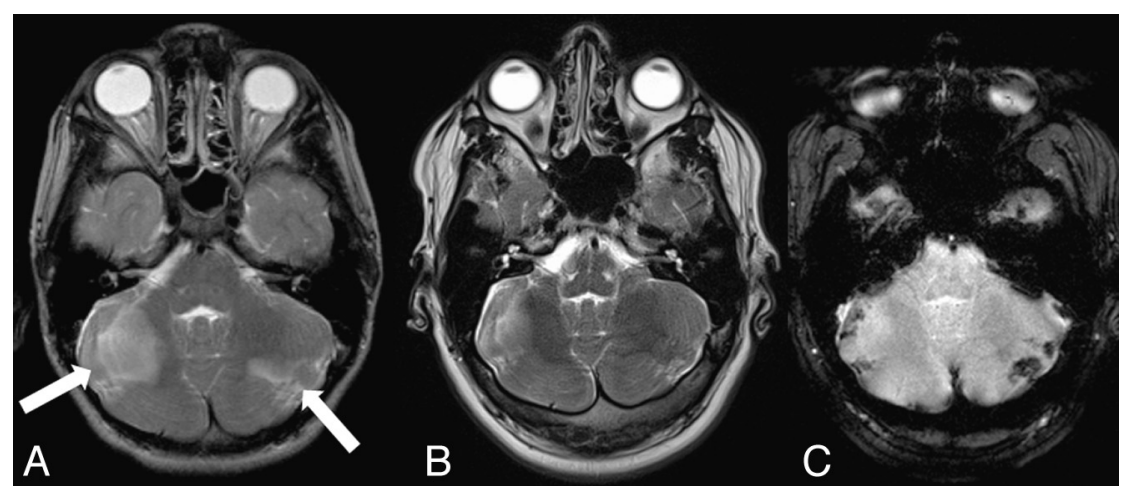

FIG 1. T2-weighted images of a patient obtained 5 years apart $(A$ and $B)$ with cerebellar tubers demonstrating decreased size and T2 signal during a 5-year period. Gradient-echo imaging $(C)$ demonstrates calcification related to the cerebellar tubers.

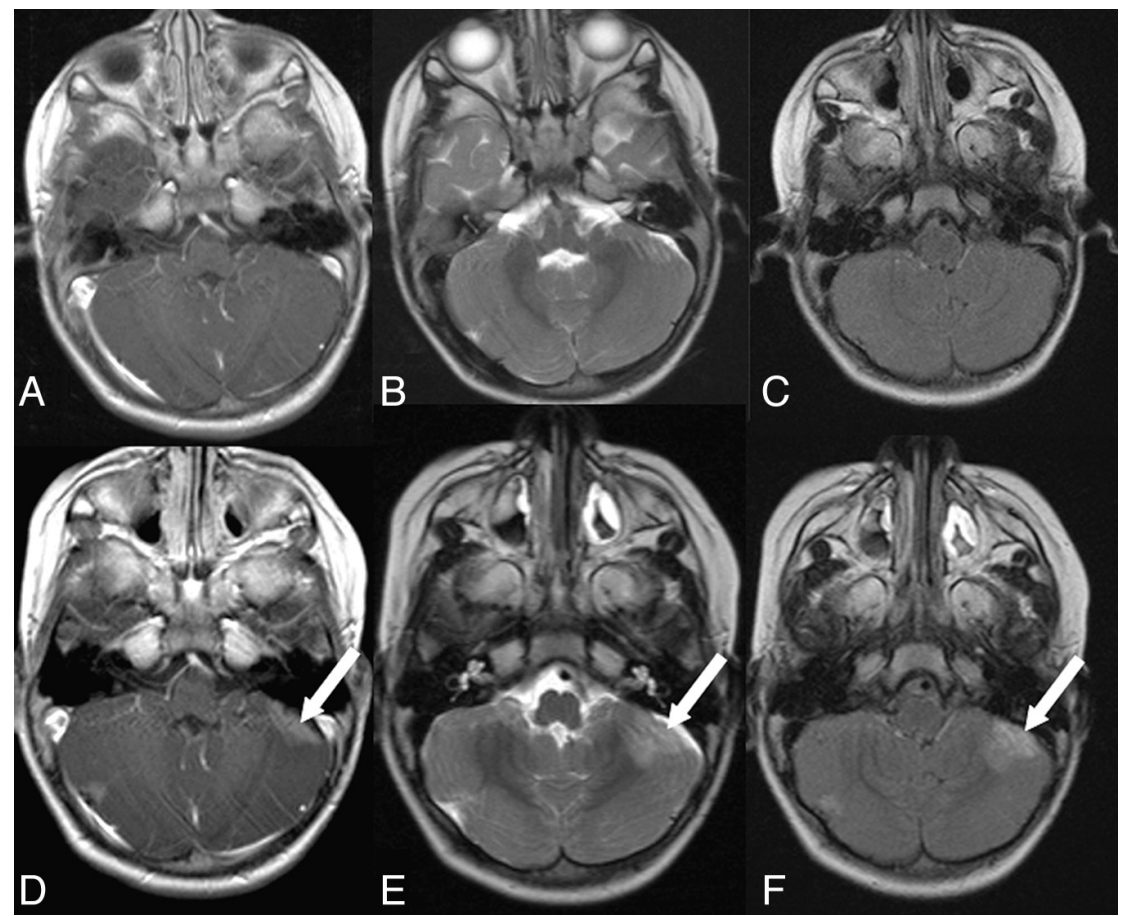

FIG 2. Images from a patient obtained 1 year apart $(A-C$ and $D-F)$ demonstrating changes in a left-sided cerebellar tuber (arrows) and a smaller right-sided tuber. Increased tuber enhancement ( $A$ and $D$ ), increased T2 signal ( $B$ and $E$ ), and new FLAIR signal abnormalities ( $C$ and $F$ ) are seen.

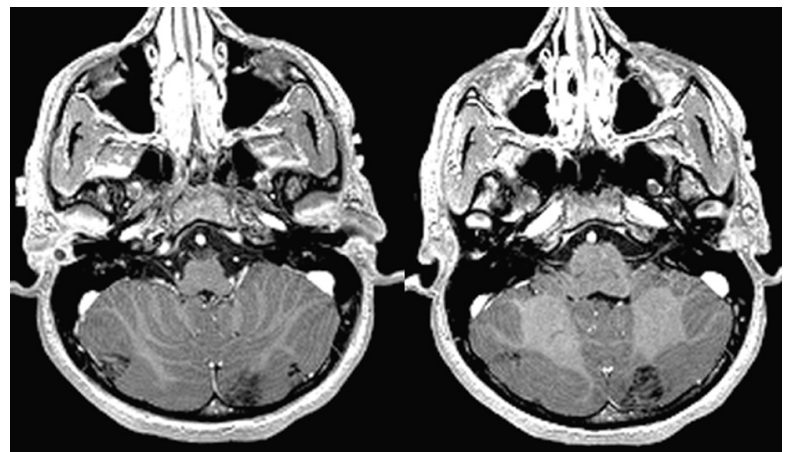

FIG 3. Images from a patient with contrast-enhancing cerebellar lesions demonstrating the "zebra" pattern of enhancement.
(17.1\%) had cerebellar atrophy. In the patients without cerebellar tubers, 6 of 110 (5.5\%) had cerebellar atrophy (Fig 4). There were a total of 16 SGAs among the 145-patient cohort. There were 9 SGAs in the 110 patients without cerebellar tubers $(8.2 \%)$ and 7 in the 35 patients with cerebellar tubers $(20.0 \%)$.

\section{DISCUSSION}

We found that cerebellar tubers were present in $24.1 \%$ of our 145 pediatric and young adult TSC cohort, similar to the percentage in a recent report of one-third of 27 patients with TSC having cerebellar abnormalities on MR imaging. ${ }^{7}$ Our imaging features of cerebellar tubers were similar to those in previous reports with predominantly wedge-shaped lesions that are hyperintense on T2 and hypo- or isointense on $\mathrm{T} 1 .^{7}$ Prior work reports that cerebellar tubers $(3 \%-92 \%)$ are more likely to enhance than cortical tubers $(3 \%-4 \%))^{7,11}$ This difference may result from impaired blood-brain barrier function near areas with inflammation and cortical disorganization. ${ }^{12}$ In our cohort, $51 \%$ of the cerebellar tubers enhanced, often in a "zebralike" pattern"; this frequency and pattern are similar to those in prior reports. The zebralike pattern may reflect the underlying cerebellar anatomy with interposed cerebellar CSF-filled sulci between the neuronal elements (Fig 3). Nine tubers demonstrated a change in enhancement pattern with time, and this evolving pattern may contribute to the widely differing frequencies $(3 \%-92 \%)$ among reports. ${ }^{711}$

Traditionally, tubers were considered stable lesions resulting from abnormal cortical migration during early fetal development. Recently, however, a few case reports and studies document the dynamic nature of a small percentage of tubers. ${ }^{13}$ One report documented 2 patients with cystlike tubers that increased in size and number with time. ${ }^{13}$ In our cohort, $52 \%$ of cerebellar tubers changed in some manner when longitudinally studied, supporting the dynamic nature of the infratentorial tubers. In patients with multiple cerebellar tubers, some of the tubers changed while others remained static; while in others, several tubers changed in parallel. The pathologic specimens of the resected cystlike tuber demonstrated the typical giant or balloon cells with a disorganized cortex; however, the subcortical white matter showed more striking fiber depletion and gliosis than in supratentorial tubers. ${ }^{13}$ The pathogenesis of the change in these cystlike tubers is still unknown; possibilities include disordered migration, tuber de- 


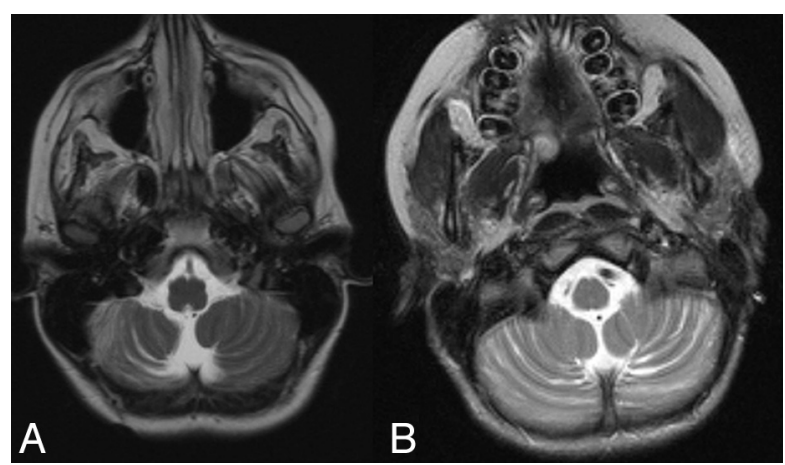

FIG 4. Global cerebellar atrophy was present in both patients with $(A)$ and without $(B)$ cerebellar tubers.

generation, accelerated astroglial apoptosis, or a combination of these processes. ${ }^{13}$ A spectrum of degenerative changes may occur in all cortical tubers, with cystlike tubers representing a subset undergoing more rapid changes. ${ }^{13}$ We lack the radiologic-pathologic correlation of cerebellar tubers but suspect a similar process as occurs in the supratentorial cystlike tubers. Previously resected cerebellar tubers have shown abnormal neuronal migration, gliosis, calcification, and folial atrophy with similar pathology as is seen in the supratentorial lesions ${ }^{10}$; this pathology is reflected in the MR imaging appearances.

Although supratentorial cortical tubers are considered responsible for the neurologic and often epileptic manifestations of TSC, many tubers appear epileptically inactive and seizures may arise from the cortex surrounding the tuber. ${ }^{14}$ Similarly, cerebellar symptoms are not clearly associated with cerebellar tubers. ${ }^{7,15}$ A correlation between autism and posterior fossa tubers was found in 1 study, while others correlated autistic symptoms (social isolation, stereotypical behavior, and communication and developmental problems) with right-sided tubers. ${ }^{4,16,17}$ However, other studies correlated autism in TSC with temporal lobe tubers ${ }^{18,19}$ and left temporal lobe epileptiform foci. ${ }^{20}$ Future studies should examine the relationship between cerebellar lesions, the severity of phenotype, and cognitive behavioral function, controlling for the effects of epilepsy and cerebral tuber burden.

The reported incidence of cerebellar atrophy varies from $4 \%$ to $13 \%$ in patients with TSC. ${ }^{5,7,15}$ Additionally, is this atrophy reflecting a primary aberrant neuronal developmental process or rather the sequela of seizures or other associated destructive processes? The similarities in the pathologic cerebellar tuber specimens to the supratentorial tuber specimens may be indicative of a similar primary abnormal developmental process. ${ }^{10}$ In our cohort, the presence of cerebellar tubers and the presence of global cerebellar atrophy were associated. Cerebellar tubers tend to calcify more frequently than cortical tubers. Moreover, 54\% of the cerebellar tubers in our cohort were at least partially calcified, with associated retraction deformity in $85 \%$. The cerebellar calcification may be a manifestation of the end stage of the abnormal neuronal organization, ${ }^{12}$ and the high frequency of tuber calcification and retraction may account for the associated focal atrophy previously reported. ${ }^{5}$ Similarly, the global cerebellar atrophy may reflect underlying abnormal gliosis or treatment-related effects.

To date, the literature is mixed on whether cerebellar tubers are associated with more phenotypically severe TSC. A relation- ship between a certain subtype of cortical tubers "type C" and a more severe phenotype has been suggested, with the "type C" tubers corresponding to the previously described cystlike tuber type. ${ }^{21}$ Clinically, these patients had difficult-to-control and more frequent seizures as well as lower intelligence quotient scores, ${ }^{21}$ and they also had a larger cortical tuber burden, a higher percentage of the TSC2 gene mutation, and a higher occurrence of SGAs. ${ }^{21}$ There was, however, no significant difference detected in the percentage of cerebellar lesions among the 3 cortical tuber groups, again raising questions of whether cerebellar lesions and SGAs are related and whether either are indicative of a more severe disease subset. Cerebellar tubers are not reported to occur in the absence of cortical tubers, and patients with cerebral and cerebellar tubers have significantly more global cortical lesions than patients without cerebellar tubers. ${ }^{5}$ In a future study, we would like to correlate our findings in the cerebellum with our cohort's supratentorial cortical tuber burden and clinical phenotype.

There are limitations to our study, including the retrospective nature, the limited follow-up examinations available for some of our patients, and the potential for bias, because our epilepsy center is a large TSC referral center and may, therefore, have a TSC patient population with a more severe phenotype than average. We also recognize that if, in fact, the presence of cerebellar lesions is associated with more phenotypically severe TSC, then our reported incidence of lesion change could be an overestimate because these patients could have undergone more frequent scanning because of their more active disease.

\section{CONCLUSIONS}

We think that this study of 145 young patients with TSC documents the high prevalence of posterior fossa tubers as well as highlights the previously unstudied great number with a dynamic longitudinal imaging appearance. A higher percentage of patients with cerebellar lesions developed SGAs than patients with TSC without cerebellar lesions. Because this is the first reported longitudinal study of cerebellar lesions in TSC, further investigation may provide additional insight into TSC pathology and clinical manifestations of the disease with time.

Disclosures: Orrin Devinsky—UNRELATED: Board Membership: Epilepsy Therapy Project, Comments: dedicated to translational research in epilepsy (no compensation provided); Other: planning to apply for an National Institutes of Health grant on Everolimus in TSC and electrophysiology.

\section{REFERENCES}

1. Baskin HJ. The pathogenesis and imaging of the tuberous sclerosis complex. Pediatr Radiol 2008;38:936-52

2. Dabora SL, Jozwiak S, Franz DN, et al. Mutational analysis in a cohort of 224 tuberous sclerosis patients indicates increased severity of TSC2, compared with TSC1, disease in multiple organs. Am J Hum Genet 2001;68:64-80

3. Prather P, de Vries PJ. Behavioral and cognitive aspects of tuberous sclerosis complex. J Child Neurol 2004;19:666-74

4. Eluvathingal TJ, Behen ME, Chugani HT, et al. Cerebellar lesions in tuberous sclerosis complex: neurobehavioral and neuroimaging correlates. J Child Neurol 2006;21:846-51

5. Martí-Bonmatí L, Menor F, Dosdá R. Tuberous sclerosis: differences between cerebral and cerebellar cortical tubers in a pediatric population. AJNR Am J Neuroradiol 2000;21:557-60

6. O'Callaghan FJ, Harris T, Joinson C, et al. The relation of infantile 
spasms, tubers, and intelligence in tuberous sclerosis complex. Arch Dis Child 2004;89:530-33

7. Ertan G, Arulrajah S, Tekes A, et al. Cerebellar abnormality in children and young adults with tuberous sclerosis complex: MR and diffusion weighted imaging findings. J Neuroradiol 2010;37:231-38

8. Chu-Shore CJ, Frosch MP, Grant PE, et al. Progressive multifocal cystlike cortical tubers in tuberous sclerosis complex: clinical and neuropathologic findings. Epilepsia 2009;50:2648-51

9. Lee A, Maldonado M, Baybis M, et al. Markers of cellular proliferation are expressed in cortical tubers. Ann Neurol 2003;53:668-73

10. Jay V, Edwards V, Musharbash A, et al. Cerebellar pathology in tuberous sclerosis. Ultrastruct Pathol 1998;22:331-39

11. Grajkowska W, Kotulska K, Jurkiewicz E, et al. Brain lesions in tuberous sclerosis complex: review. Folia Neuropathol 2010;48: 139-49

12. Castillo M, Whaley RA, Point SW, et al. Gyriform enhancement in tuberous sclerosis simulating infarction. Radiology 1992;185: 613-14

13. Chu-Shore CJ, Major P, Montenegro M, et al. Cyst-like tubers are associated with TSC2 and epilepsy in tuberous sclerosis complex. Neurology 2009;72:1165-69

14. Major P, Rakowski S, Simon MV, et al. Are cortical tubers epilepto- genic? Evidence from electrocorticography. Epilepsia 2009;50: $147-54$

15. Menor F, Marti-Bonmati L, Mulas F, et al. Neuroimaging in tuberous sclerosis: a clinicoradiological evaluation in pediatric patients. Pediatr Radiol 1992;22:485-89

16. Weber AM, Egelhoff JC, McKellop JM, et al. Autism and the cerebellum: evidence from tuberous sclerosis. J Autism Dev Disord 2000;30:511-17

17. Asano E, Chugani DC, Muzik O, et al. Autism in tuberous sclerosis complex is related to both cortical and subcortical dysfunction. Neurology 2001;57:1269-77

18. Bolton PF, Griffiths PD. Association of tuberous sclerosis of temporal lobes with autism and atypical autism. Lancet 1997;349:392-95

19. Bolton PF, Park RJ, Higgins NP, et al. Neuro-epileptic determinants of autism spectrum disorders in tuberous sclerosis complex. Brain 2002;125:1247-55

20. Numis AL, Major P, Montenegro MA, et al. Identification of risk factors for autism spectrum disorders in tuberous sclerosis complex. Neurology 2011;76:981

21. Gallagher A, Grant EP, Madan N, et al. MRI findings reveal three different types of tubers in patients with tuberous sclerosis complex. J Neurol 2010;257:1373-81 